\title{
Demonstrating Tangential Velocity by a Self-Coded Robot
}

\author{
Pete Hwang ${ }^{1}$ and Danilo JR Tadeo* \\ ${ }^{1}$ Yongsan International School of Seoul, Seoul, Korea \\ "Advisor
}

\section{$\underline{\text { ABSTRACT }}$}

Tangential velocity is a concept that is often taught as a part of rotation and is the linear velocity an object has as it turns about a fixed axis. The main goal of this research project was to examine this concept and demonstrate it on a self-coded robot. The robot was designed with two rotating motors with two wings of different lengths to be attached on top, and the motors were connected to a circuit board and coded using the programming language $\mathrm{C}$ to rotate with equal and constant angular velocities upon initiation. The main hypothesis is that the length of rotating wings is directly proportional to the tangential velocity of the wings. The project provides a new way of demonstrating tangential velocity in a high school physics class.

\section{Background of the Study}

One of the first areas of study in Physics is translational motion that concerns the motion of an object along a straight line. During this period, students cover relatively intuitive terms such as position (s), velocity (v), acceleration (a), or time (t). Another type of motion is rotational motion in which an object turns about an axis. However, the connection from translation to rotation is quite large, and many students find it difficult to grasp at first. Reasons vary, but generally new symbols and unfamiliarity with the concepts are the main culprits behind the challenge (Halliday and Resnick, 2014). For instance, terms such as angular position $(\theta)$, which is the angle in radians (rad), of a reference line relative to a fixed position; angular velocity $(\omega)$, which is the change in the angular position over time; and the angular acceleration $(\alpha)$, which is the change in the angular velocity over time are introduced in this section. One way to resolve these difficulties is through classroom demonstrations that allow students a laboratory-like experience within the classroom, by facilitating hypothesis, testing and observation of results pertaining to a physical phenomenon (Hillel, 2005).

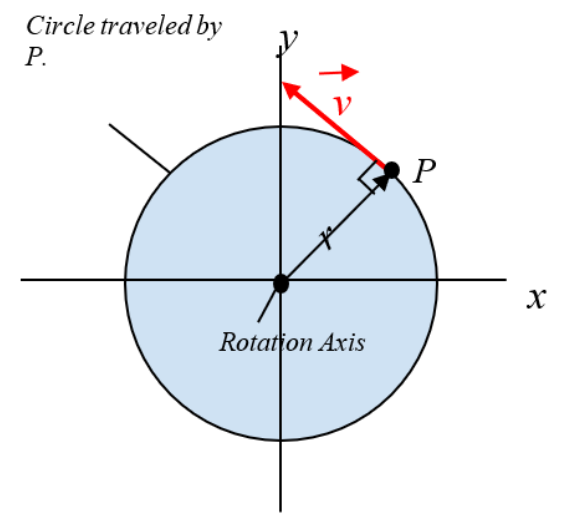

Figure 1. Velocity vector of a point distance (r) from the axis of rotation. 
The general equation for tangential velocity, $v=r \omega$ with the SI unit of $\mathrm{m} / \mathrm{s}$ can be derived using Figure 1 above. The perpendicular distance is the distance between a point $P$ and the axis of rotation measured along a perpendicular to the axis, which in this case is the velocity vector of $P$. When one component remains constant, such as $\omega$, and the other altered, such as $r$, the effect on $V_{t}$ can be predicted. But understanding the concept of tangential velocity can also be challenging since there are only a few demonstrations to show this, i.e., the wheel labeled with strips of paper at different radii from the center of rotation (Youtube, accessed on May 2021).

This paper presents a new tool to demonstrate the concept of tangential velocity that is very essential in connecting the translation and rotational motion. This tool is a self-coded robot that is designed to exhibit that the length (perpendicular distance, $r$ ) of the rotating wings is proportional to the tangential speed of the motor wings. The self-coded robot may be added to the few physical demonstrations available to aid students' understanding of rotational motion.

\section{Methodology}

\section{Robot Making}

The parts of the robots were obtained from the ROBOKIT Step 1 package provided by the RoboRobo ${ }^{1}$ robotics company in South Korea. As portrayed in Figure 2, the robot was mainly designed to maximize the space between the two motors. The dimensions of the main body of the robot is $28.0 \times 8.0 \mathrm{~cm}$. Two wings of different length were attached on top of the motors. Wing 1 was created to be significantly longer than wing 2 having the lengths of $23.7 \mathrm{~cm}$ and 7.7 $\mathrm{cm}$ respectively.

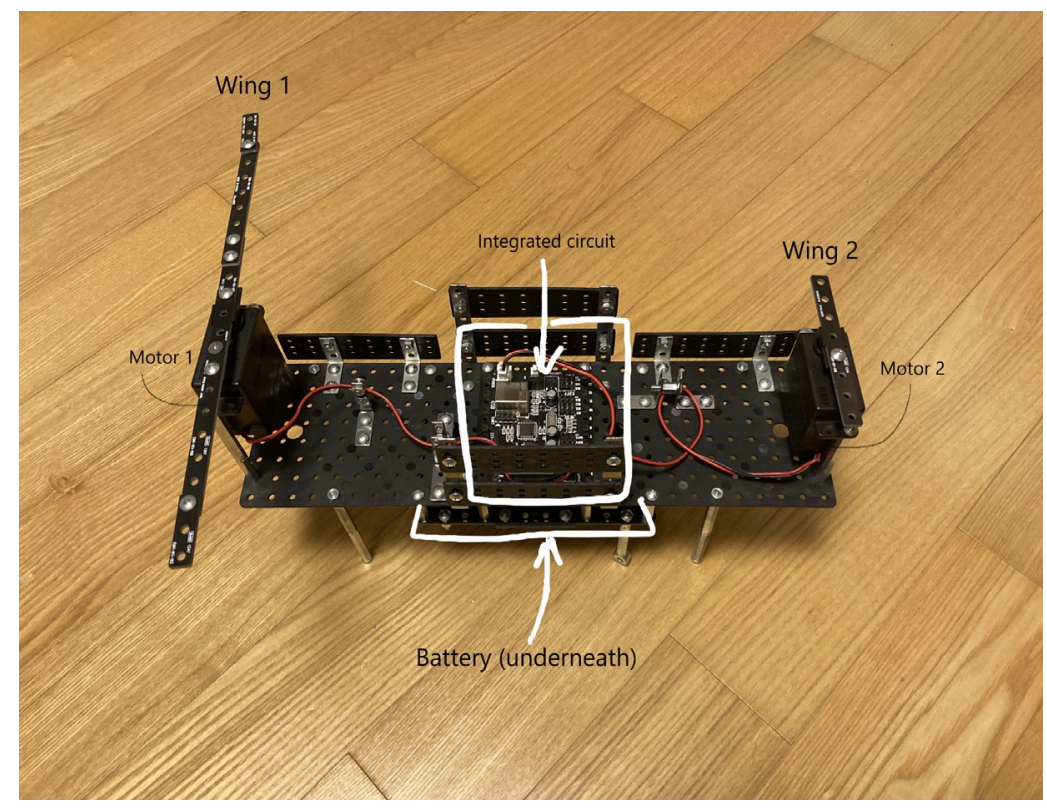

Figure 2. The self-coded robot that exemplifies tangential velocity

\footnotetext{
${ }^{1}$ Roborobo is a company specializing in cultivating talents which lead the future of industrial revolution.
} 


\section{Coding}

For the programming part, a CPU included in the ROBOKIT Step 1 package was utilized and the appropriate programming application was downloaded from the internet. The plan was to start the motors when the robot is turned on and stop them when turned off. To implement this functionality, a simple infinite loop was written to run when the machine was turned on, running the motors until the machine was turned off, which would stop the loop. The rotational speeds for the motors were set to equal and constant values which would satisfy the experimental purpose of testing the effect of the length of wings on tangential velocity.

\section{Discussion}

This section presents the two main components that were taken into consideration when designing the robot: stabilization and space efficiency.

Initially, issues arose when the wings were colliding with one another due to their proximity in height and length and establishing stability in the structure. This was solved by increasing the length of the robot and lowering the height of one of the motors. Metal legs were attached accordingly to also ensure stability. As shown in Figure 2, the robot is designed in a long rectangular fashion, which puts the two motors and wings at a maximum distance from one another.

Another issue was space efficiency. Once the collision issue was eliminated the next problem was working with the limited space on the top. The two components to manage were the integrated circuit board and the battery pack powering the motors. If both components were stacked on top of each other, this would pose risks of collisions with the rotating wings and difficulties when changing the batteries later. Increasing the height of the motors was a possible solution, but this placed a potential risk in the overall stability of the structure. As such, the battery pack was placed underneath the robot.

\section{Conclusion}

In conclusion, this project was a major success. As predicted, the longer wing appeared to rotate faster than the shorter wing despite their equal angular speeds. This is because the longer wing is able to cover a greater circumference per second than the shorter wing, producing the effect of rotating faster.

A few areas of reconsideration would be the features of the robot. As of now, the angular or tangential velocities of the motors cannot be seen nor recorded in a timely manner. An additional function that can be added would be collecting data. Speed sensors can be implemented to log the two types of velocities for both motors. In addition, wings of more purposeful dimensions can be constructed. Currently, the shorter wing is $7.7 \mathrm{~cm}$ and the longer 23.7 $\mathrm{cm}$. When collecting data, it is more efficient to keep any changes to the variables consistent. For instance, the lengths could differ by a factor of 2 or 3 . For instance, $7.7 \mathrm{~cm}$ v.s $14.4 \mathrm{~cm}$ or $7.7 \mathrm{~cm}$ v.s. $23.1 \mathrm{~cm}$.

This robot can definitely be used in a physics classroom as an alternative way to demonstrate the concept of tangential velocity. In contrast to the wheel labeled with strips of paper at different radii from the axis of rotation, this robot manages to isolate the two radii and demonstrate the effect directly. Combining different fields of academics, such as computer science and physics, is something that students should be practicing in this day and age, as it helps to solidify the knowledge learned from both areas. If given the chance, high school students should take the opportunity to create these projects to boost their comprehension of challenging concepts.

\section{Acknowledgments}

I would like to thank my advisor Danilo Tadeo for helping me with this project. 


\section{References}

Hillel, J. (2005). Physics education research: A comprehensive study. Toronto, ON: University of Toronto.

RoboRobo. Roborobo. (n.d.). https://eng.roborobo.co.kr/company/overview.

Walker, J., Halliday, D., \& Resnick, R. (2014). In Fundamentals of physics: extended (10th ed., pp. 258-259). essay, Wiley.

YouTube. (2017). Angular speed vs linear speed. YouTube. https://www.youtube.com/watch?v=3Lape_vG0Sc. 